D.O.I. $10.3895 / \mathrm{S} 1808-04482008000200006$

\title{
ARQUITETURAS DE REFERÊNCIA PARA REDES DE COOPERAÇÃO EM SISTEMAS PRODUTIVOS DINÂMICOS SEGUNDO A ABORDAGEM EKD (ENTERPRISE KNOWLEDGE DEVELOPMENT)
}

\section{REFERENCE ARCHITECTURES FOR DYNAMIC PRODUCTIVE SYSTEMS COOPERATION NETWORKS CONSIDERING ENTERPRISE KNOWLEDGE DEVELOPMENT (EKD) APPROACH}

\author{
Gilberto Miller Devós Ganga ${ }^{1}$; Fábio Muller Güerrini ${ }^{2}$; Luiz Cesar Ribeiro Carpinetti ${ }^{3}$ \\ ${ }^{1}$ São Paulo State University - UNESP - Tupã - Brasil giba@tupa.unesp.br \\ ${ }^{2}$ University of São Paulo - EESC/USP - São Carlos - Brasil guerrini@sc.usp.br \\ ${ }^{3}$ University of São Paulo - EESC/USP - São Carlos - Brasil carpinet@,sc.usp.br
}

\begin{abstract}
Resumo
A pressão por menores ciclos produtivos, menores tempos de lançamentos de produtos, maior variedade do mix de produtos e inovações tecnológicas, entre outros, tornou imperativo a busca ou desenvolvimento de novas configurações organizacionais que pudessem suportar o atual ambiente complexo e dinâmico. As redes de cooperação, principalmente de pequenas e médias empresas, tornaram-se então uma alternativa viável para estas se manterem competitivas no mercado. Nesse sentido, uma arquitetura de referência pode fornecer elementos para a integração e coordenação entre empresas. Este trabalho pretende apresentar os conceitos relativos à arquitetura de referência para redes entre empresas constituídas em sistemas produtivos dinâmicos como forma para coordenar recursos, capacidades e competências. Pretende-se também propor um modelo genérico e conceitual de arquitetura de referência e relacioná-lo a abordagem EKD (Enterprise Knowledge Development). Como conclusão geral, observa-se a grande aplicabilidade da metodologia EKD como uma Arquitetura de Referência ou até mesmo suportando outra arquitetura comercial existente.
\end{abstract}

Palavras-chave: redes de cooperação; modelagem de redes; arquitetura de referência; empresas virtuais; enterprise knowledge development (EKD).

\section{Introdução}

Num ambiente cada vez mais complexo e dinâmico é necessário que as empresas consigam projetar um modelo organizacional cada vez mais integrado no sentido de maximizar intensivamente a utilização dos recursos da mesma, gerando assim maiores níveis de flexibilidade que suportem as mudanças constantes e consequentemente elevem o nível de competitividade da 
empresa como um todo.

Nesse sentido, a comunicação, mais especificamente o uso estratégico da informação, é um mecanismo fundamental na integração organizacional, através da coordenação dos diferentes departamentos, compondo assim processos de negócios que estejam alinhados á estratégia global da organização como um todo.

No entanto, desenvolver uma estrutura organizacional integrada não significa simplesmente adotar um sistema de informação. É necessário que se desenvolva um modelo, que reconheça as estratégias da empresa, seus processos de negócios e posteriormente se traduza em um modelo ou software computacional que apóie esta estratégia e os processos que a suportam. Esse modelo de referência pode ser denominado "arquiteturas de referência".

Porém, o objeto maior, a integração, como forma de incremento à competitividade, não se restringe mais somente ao nível organizacional de uma empresa. A fim de maximizar os resultados, compartilhar riscos e aumentar a capacidade de inovar tecnologicamente novos arranjos organizacionais têm se formado a partir da cooperação entre empresas formando redes de cooperação nesse sentido.

Contudo, o desempenho dessas redes de cooperação depende do grau de coordenação entre os agentes que compõem essa nova estrutura. Nesse sentido, o desenvolvimento de arquiteturas de referência para essas redes de cooperação torna-se um fator crucial no estabelecimento de um framework comum, ou seja, uma mesma plataforma que permita o compartilhamento de recursos, capacidades e competências entre os agentes que compõem a rede a fim de aumentar a competitividade dos mesmos.

A proposição de uma arquitetura de referência para redes de cooperação pode ser ainda mais complexa se considerarmos o fato da rede ser formada por pequenas empresas. Segundo Chalmeta; Campos e Grangel (2001) a maioria das arquiteturas de referência são desenvolvidas para grandes empresas. Uma arquitetura de referência projetada para redes de cooperação entre pequenas empresas pode torna-se um fator estratégico no suporte a formação, desenvolvimento e dissolução da rede.

Dado o grau de informalidade das pequenas empresas (o que de certa maneira diminui os custos de transação), torna-se imprescindível essa arquitetura de referência para promover a agilidade entre os agentes como um desafio em responder às mudanças e utilizar essa capacidade de resposta como estratégia competitiva.

Assim, este trabalho pretende apresentar os conceitos relativos à arquitetura de referência para redes entre empresas constituídas em sistemas produtivos dinâmicos como forma para coordenar recursos, capacidades e competências. Pretende-se também propor um modelo genérico e 
conceitual de arquitetura de referência e relacioná-lo a abordagem EKD (Enterprise Knowledge Development).

\section{Redes como fator de transferência de inovações tecnológicas}

Segundo o Manual de Oslo (2004), o campo das políticas de inovação são definidos através de quatro níveis de desagregação:

1. As condições estruturais mais amplas dos fatores institucionais nacionais (fatores jurídicos, econômicos, financeiros e educacionais);

2. A base da ciência e engenharia: conhecimento acumulado e instituições de ciência e tecnologia que sustentam a inovação comercial;

3. Fatores de transferência: são os que influenciam fortemente a eficácia dos elos de fluxo de informações e competências e absorção de aprendizado, essenciais para a inovação comercial; e

4. Dínamo da inovação é o domínio mais central da inovação comercial, cobrindo fatores dinâmicos dentro das empresas ou em sua vizinhança imediata que têm um impacto muito direto em sua capacidade inovadora.

Nesse sentido, os elos cooperativos entre empresas são considerados fatores de transferência de inovação. E quanto maior o grau de cooperação entre esses elos, maior a necessidade de coordenação (CAMARINHA-MATOS e AFSARMANESH, 1999).

Para Rycroft e Kash (2004) as redes de inovação autoorganizadas, formadas a partir de diferentes empresas, são capazes de combinar novos conhecimentos científicos e tecnológicos e recombinar velhas competências sem a intervenção de um controlador central. O termo "autoorganizadas" surge a partir da iniciativa dos indivíduos (organizações) que assumem espontaneamente a iniciativa de utilizar uma infra-estrutura global para estabelecer os relacionamentos necessários a fim de atingir seus objetivos de negócios.

Nesse cenário, um fator preponderante na proliferação das redes de inovação tecnológicas tem sido a globalização. A internacionalização cada vez maior dos negócios (recursos) vêm forçando as organizações à cooperarem a fim de aumentar sua competitividade. A base para as redes de inovação tecnológicas é o compartilhamento de conhecimento, configurando então, "redes de aprendizagem organizacional", baseadas em níveis crescentes de confiança mútua, que culminam num número crescente de inovações tecnológicas entre as organizações que compõem a rede (RYCROFT e KASH, 2004).

O aprendizado é a chave a para a inovação. A capacidade da rede aprender e se adaptar às 
condições econômicas e tecnológicas vai determinar a competitividade da mesma. O aprendizado é cumulativo e os mercados sozinhos não são suficientes para selecionar o aprendizado ótimo. Nesse sentido, a seleção do aprendizado crítico para inovação tecnológica deve ser feita, portanto, autoconscientemente pelas organizações, fazendo a inovação a partir do compartilhamento da informação e formação de parcerias com novas competências para resolver problemas tecnológicos ou alavancar oportunidades (RYCROFT e KASH, 2004).

Outra razão chave das redes de inovação é a capacidade de aprender e se auto-organizar para que elas desenvolvam confiança mútua e relacionamentos informais (RYCROFT e KASH, 2004).

Deste modo, uma arquitetura de referência projetada para a rede pode alavancar esse compartilhamento de recursos, capacidades e competências entre os agentes e promover competitividade por meio das inovações geradas nesse processo.

\subsection{Vertentes Teóricas de Redes}

Para Sacomano Neto e Truzzi (2004) a análise da configuração das redes recebe contribuições da sociologia econômica, da teoria das organizações e do novo institucionalismo. Segundo os autores o nível de análise das redes tem se consolidado como uma abordagem adequada para a compreensão do binômio competição/cooperação que permeia os mercados e as relações entre os atores econômicos. Nesse sentido faz-se necessário neste trabalho descrever algumas perspectivas teóricas contemporâneas em análise organizacional, a saber: Ecologia das Populações, Dependência de Recursos, Contingëncia Estrutural, Nova Economia Institucional e Novo Institucionalismo (SACOMANO NETO e TRUZZI, 2002).

O Quadro1, proposto por Sacomano Neto e Truzzi (2002) caracteriza alguns elementos em relação ás perspectivas teóricas contemporâneas em análise organizacional. 
Quadro 1 Caracterização de alguns elementos acerca das novas perspectivas teóricas

\begin{tabular}{|c|c|c|c|}
\hline \multirow[b]{2}{*}{ PERSPECTIVA } & \multicolumn{3}{|l|}{ VARIÁVEIS } \\
\hline & Nível de análise & Principais Variáveis & Principais críticas \\
\hline Ecologia das Populações & $\begin{array}{l}\text { População de } \\
\text { organizações } \\
\text { Nichos ecológicos }\end{array}$ & $\begin{array}{l}\text { Variação, seleção e } \\
\text { retenção de populações } \\
\text { de organizações }\end{array}$ & $\begin{array}{l}\text { Dificuldade de } \\
\text { especificar a fonte de } \\
\text { variação ambiental } \\
\text { Ignoram os processos } \\
\text { gerenciais }\end{array}$ \\
\hline $\begin{array}{l}\text { Dependência de } \\
\text { Recursos }\end{array}$ & $\begin{array}{l}\text { Interações ambientais } \\
\text { Controles } \\
\text { interorganizacionais }\end{array}$ & $\begin{array}{l}\text { Fluxo de Recursos } \\
\text { Ação organizacional no } \\
\text { ambiente }\end{array}$ & $\begin{array}{l}\text { Limitação da ação } \\
\text { organizacional } \\
\text { O que dirige a ação } \\
\text { gerencial }\end{array}$ \\
\hline Contingência Estrutural & $\begin{array}{l}\text { Fatores ambientais que } \\
\text { condicionam a forma } \\
\text { organizacional }\end{array}$ & $\begin{array}{l}\text { Decisões estratégicas } \\
\text { contingentes às pressões } \\
\text { ambientais }\end{array}$ & $\begin{array}{l}\text { Demasiada crença na } \\
\text { capacidade adaptativa das } \\
\text { organizações } \\
\text { Dificuldade de apresentar } \\
\text { respostas racionais as } \\
\text { pressões internas e } \\
\text { externas }\end{array}$ \\
\hline $\begin{array}{l}\text { Nova Economia } \\
\text { Institucional }\end{array}$ & $\begin{array}{l}\text { Custos de transação e } \\
\text { formas de governança }\end{array}$ & $\begin{array}{l}\text { Intercâmbio de bens e } \\
\text { serviços em um mercado } \\
\text { livre }\end{array}$ & $\begin{array}{l}\text { Negligentes quanto à } \\
\text { estruturas e lutas de } \\
\text { poder } \\
\text { Interesses individuais e } \\
\text { grupais derivados de uma } \\
\text { estrutura de interesses }\end{array}$ \\
\hline Novo Institucionalismo & $\begin{array}{l}\text { Como as organizações } \\
\text { surgem, tornam-se } \\
\text { estáveis e são } \\
\text { transformadas } \\
\text { Estruturação da ação e } \\
\text { ordem de significado }\end{array}$ & $\begin{array}{l}\text { Aspectos coercitivos, } \\
\text { normativos e cognitivos }\end{array}$ & $\begin{array}{l}\text { Determinisno } \\
\text { institucional para a } \\
\text { difusão das formas } \\
\text { organizacionais } \\
\text { Dificuldade de } \\
\text { mensuração das variáveis }\end{array}$ \\
\hline
\end{tabular}

Fonte: Adaptado de Sacomano Neto e Truzzi (2002)

A próxima seção tratará de maneira sintética alguns conceitos sobre estrutura de governança, teoria dos custos de transação nas relações interorganizacionais e fundamentalmente a questão da confiança como instrumento de fortalecimento desses relacionamentos.

\subsection{Estruturas de Governança e Custos de Transação nas Relações Interorganizacionais e a Questão da Confiança}

As relações interorganizacionais se referem a qualquer tipo de contatos entre duas ou mais organizações, variando da forma concorrencial e antagônica para aquelas de natureza cooperativa, tanto entre organizações similares ou diferentes, envolvendo transações, fluxos e ligações de recursos relativamente duradouros (OLIVER, 1990 apud CUNHA e MELO, 2006). 
A existência de contatos inter-relacionais implica que as organizações envolvidas desenvolvam processo de escolha do grupo de interação com aqueles que são relevantes no conjunto de organizações (RING e VAN DE VEN, 1994 apud CUNHA e MELO, 2006).

Entre esses relacionamentos, percebe-se um aumento da importância das relações entre empresas visando à colaboração como instrumento estratégico (CUNHA e MELO, 2006).

Nesse contexto das relações inter-organizacionais como diferencial estratégico é interessante contextualizar a economia dos custos de transação e as estruturas de governança.

A importância de se analisar as alianças inter-organizacionais como um processo é a de que os agentes das organizações precisam conhecer, além das condições de investimentos, os tipos de estrutura de governança requeridos para um relacionamento. A forma como se negociam, executam e modificam os termos de um relacionamento influencia fortemente o julgamento das partes, se as ações tomadas no relacionamento são eqüitativas e eficientes, além de influenciar na motivação para a continuação ou término do relacionamento" (RING E VAN DE VEN, 1994 apud CUNHA e MELO, 2006).

A caracterização da eficiência de um determinado sistema produtivo não depende apenas da identificação de quão bem cada um de seus segmentos equaciona seus problemas de produção. Quanto mais apropriada for a coordenação entre os componentes do sistema, menores serão os custos de cada um deles, mais rápida será a adaptação às modificações de ambiente e menos custosos serão os conflitos inerentes às relações cliente-fornecedor (AZEVEDO, P., 2000).

A coordenação não é uma característica intrínseca dos sistemas produtivos, mas sim, uma construção dos agentes econômicos. Com a finalidade de reduzir custos de transação (custos de elaboração e negociação de contratos; custos de mensuração e físcalização dos direitos de propriedade; custos de monitoramento de desempenho; custos relativos à organização de atividades; e custos de problemas de adaptação), os agentes fazem uso de mecanismos apropriados para regular determinada transação, denominados estruturas de governança (WILLIAMSON, 1985 apud AZEVEDO, P., 2000).

O termo governança foi inicialmente utilizado por diversos autores (WILLAMSON,1985; HOLLINGSWORTH e LINDBERG, 1986; JESSOP, 1998; HUMPHREY e SCHMITZ, 2000 apud CARVALHO, 2005), para designar o processo de coordenação doa atores econômicos, nas esferas pública e privada e nos níveis local e global.

Fleury e Fleury (2000) apud Carvalho (2005) ressaltam que a idéia central da análise das cadeias de produção é a identificação das estruturas de poder ou governança em que uma ou mais empresas coordenam e controlam atividades econômicas geograficamente dispersas.

Segundo Powell (1990) e Cooper e Slagmulder (2004) a decisão de formação de redes de empresas é pouco influenciada pelos custos de transação. A economia dos custos de transação 
permite classificar as estruturas de governança como uma função das características de transação, mas não auxilia na compreensão da natureza dos relacionamentos.

Quando a estrutura de governança para monitorar os custos de transação são necessários, mas as transações envolvidas são muito pequenas ou não ocorrem com muita freqüência para justificar os custos consideráveis de um esquema bilateral de governança, pode ser mais eficiente subcontratar um terceiro para realizar a arbitragem. Esta parte deve ter a confiança de ambos os protagonistas, tanto na competência, quanto na intenção de julgamento imparcial (NOTEBOOM, 1999).

A menção ao termo confiança, citado acima, nos remete uma fator de extrema importância na formação de redes inter-organizacionais.

Para Grandori e Soda (1995) apud Cunha e Melo (2006), a confiança é um dos mais importantes e freqüentes conceitos mencionados na conexão das relações cooperativas entre firmas principalmente quando estão envolvidos processos de aprendizagem e inovação (DODGSON, 1993 apud CUNHA e MELO, 2006).

O estabelecimento da confiança entre parceiros na formação e manutenção das alianças pode reduzir os custos de coordenação e a necessidade de controles hierárquicos, o que tende a aumentar a flexibilidade da organização e a aumentar sua capacidade de se adequar às novas necessidades (ALTER e HAGE, 1993 apud CUNHA e MELO, 2006).

Para Nooteboom (2003) o fator confiança é extremamente necessário no sentido de tentar equilibrar a relação existente entre colaboração e os conflitos de interesses. Ela ainda pode influenciar no grau de aprendizado da rede como um todo, ou seja, quanto maior a sinergia devido ao aumento de confiança entre os agentes, menores os custos de transação e consequentemente maior a flexibilidade da rede na resolução de problemas, resultando assim em maior capacidade de resposta junto ao ambiente.

\subsection{Caracterização das Relações Interorganizacionais}

Para Leon (1998) apud Olave e Amato Neto (2001), as redes de empresas são formadas inicialmente com o objetivo de reduzir incertezas e riscos, organizando atividades econômicas a partir da coordenação e cooperação entre empresas.

De acordo com Amato Neto (2005, p.17-18) “as redes interorganizacionais constituem-se, portanto, em uma alternativa quanto à forma de organizar a produção de bens e serviços e podem ser utilizadas pelas empresas na busca de melhoria de sua posição competitiva. Ressalta-se, ainda, que o essencial neste conceito de redes é a figura da empresa-centro ( $h u b$-firm), ou ainda empresamãe (no caso de um complexo industrial), em torno da qual se constrói toda a rede." 
A formação de redes dinâmicas de cooperação constituem um fator fundamental de competitividade principalmente para as pequenas empresas que não detêm todas as etapas da cadeia de valor. Geralmente as grandes empresas possuem condições suficientes para dominar todas as etapas da cadeia de valor (suprimentos, logística, P\&D, produção e marketing), sendo essa uma vantagem competitiva, à medidas que estas etapas esteja, todas integradas e focadas no cliente (AMATO NETO, 2005).

Para Ribault et al. (1995) apud Olave e Amato Neto (2001), as principais vantagens da formação de redes de empresas podem ser:

- Cada uma das empresas de uma rede pode aprofundar sua especialização. É ao nível do conjunto de rede que se faz a perenidade de todo o Know how das atividades;

- As empresas de uma rede podem deste modo tornar-se o reflexo da atividade econômica dessa rede. Esta é uma forma de colocar em prática o modelo da cadeia de valor de Porter;

- As empresas escolhem-se por afinidade. Podem constituir uma rede profundamente original relativamente às empresas concorrentes, conferindo a si próprias e grau elevado de exclusividade.

O desenvolvimento das relações interorganizacionais (IOR's), tem como objetivos: transferência, intercâmbio, desenvolvimento ou produção de tecnologias; desenvolvimento de novos materiais; produtos ou informação. As relações não necessariamente implicam em ganhos monetários, mas poderá influenciar o desenvolvimento sócio-econômico de uma região, preservar a função ecológica e aumentar a qualidade de vida, dos indivíduos pertencentes à rede (GOEDERT, 2005).

A caracterização das relações interorganizacionais pode ser realizada tendo como base as seguintes variáveis:

- Ambiente;

- Mofologia;

- Tipologia; e

- Identificação das competências

A caracterização das relações interorganizacionais pode ser feita sob a ótica da formalização, centralização, direção, flexibilidade, fronteiras, tipos de inter-relações empresariais e dureza. 
Neste trabalho procurou-se dar mais ênfase às variáveis Tipologia e Morfologia de redes, já que será necessário uma melhor descrição destas em relação ao modelo de arquitetura de referência proposta.

A morfologia de redes baseia-se na identificação de nós (empresas ou atividades), posições (estrutura e divisão do trabalho), ligações (relacionamentos entre empresas - aspectos qualitativos) e fluxos (de bens: tangíveis e de informações: intangíveis) para a coordenação das transações, dos processos produtivos e da inovação no interior do sistema produtivo (BRITTO, 2002).

O estudo de tipologia de redes é importante para formalizar o tipo de relação entre empresas que será analisado indicando quais os mecanismos aplicados. As formas de redes podem ser caracterizadas por: grau de formalidade, centralização (uma empresa central exercendo governança) ou base de igualdade; e combinação de mecanismos de coordenação. As redes podem ainda ser diferenciadas quanto ao grau de centralização: simétrica (não existe empresa centralizadora), e assimétrica (uma empresa centraliza as relações).

Olave e Amato Neto (2001), apresentam no Quadro 2, a seguir, uma série de estudos sobre tipologia de redes esboçando as várias vertentes e autores.

Quadro 2 Tipologia de Redes de empresas

\begin{tabular}{|l|l|}
\hline \multicolumn{1}{|c|}{ Autor } & \multicolumn{1}{|c|}{ Tipologia } \\
\hline Grandori e Soda (1995) & $\begin{array}{l}\text { Redes Sociais: Simétricas e Assimétricas } \\
\text { Redes Burocráticas: Simétricas e Assimétricas } \\
\text { Redes Proprietárias: Simétricas e Assimétricas }\end{array}$ \\
\hline Casarotto e Pires (1998) & $\begin{array}{l}\text { Redes Top-Down: Subcontratação, terceirização, parcerias } \\
\text { Redes Flexíveis: Consórcios }\end{array}$ \\
\hline Wood Jr e Zuffo (1998) & $\begin{array}{l}\text { Estrutura Modular: Cadeia de Valor, Terceirização Atividades de Suporte } \\
\text { Estrutura Virtual: liga temporariamente rede de fornecedores } \\
\text { Estrutura Livre: de barreiras, define funções, papéis, tarefas }\end{array}$ \\
\hline Corrêa (1999); & $\begin{array}{l}\text { Rede Estratégica: desenvolve-se a partir de uma empresa que controla todas as } \\
\text { atividades } \\
\text { Rede Linear: Cadeia de valor (participantes são elos) } \\
\text { Rede Dinâmica: relacionamento intenso e variável das empresas em si. }\end{array}$ \\
\hline Porter (1998) & $\begin{array}{l}\text { Cluster: concentração territorial e geográfica de empresas. Caracterizado pelo ganho } \\
\text { de eficiência coletiva }\end{array}$ \\
\hline Bremer (1996) & $\begin{array}{l}\text { Empresa Virtual: ponto de vista Institucional (combinação das melhores } \\
\text { competências essenciais de empresas legalmente independente) e Funcional } \\
\text { concentração em competências essenciais coordenadas através de uma base de } \\
\text { tecnologia de informação) }\end{array}$ \\
\hline
\end{tabular}

Fonte: Adaptado de Olave e Amato Neto (2001). 
O Quadro 2 não tem por objetivo esgotar a análise sobre tipologia de redes, mas sim, esclarecer o mínimo possível as " $n$ " caracterizações de redes quanto suas tipologias, servindo como referencial básico na estrutura lógica deste trabalho.

\subsection{Ciclo de vidas de Redes}

Nesta seção será adotado como referência a temática das empresas virtuais por apresentarem uma maior complexidade e necessidade de agilidade no processo de formação, operação, dissolução e reconfiguração de redes de empresas.

"Na formação de redes entre empresas (PMES) existe a possibilidade destas configuraremse como redes flexíveis de pequenas e médias empresas, como clusters de empresas (agrupamentos), ou como redes de cooperação, geralmente como organizações virtuais [...]"( LEON, 1998 apud OLAVE e AMATO NETO (2001, p. 293).

Cao e Dowlatshahi (2005) definem uma empresa virtual ${ }^{1}$ como uma rede de organizações com alinhamentos temporários para explorar oportunidades de negócio. Posteriormente o alinhamento é dissolvido e os membros ficam disponíveis para outras oportunidades.

Para Amato Neto (2005), o conceito de organizações virtuais pode ser entendido, em uma primeira aproximação, como uma forma de cooperação entre empresas ou organizações, constituindo verdadeiras "redes dinâmicas de cooperação", que através da utilização das novas tecnologias da telemática (a Internet, por exemplo), alavancam a competitividade dos parceiros desta rede e possibilitam a exploração de novo oportunidades de mercado em âmbito global.

Podem se observadas as seguintes características nas empresas virtuais (CAMARINHAMATOS e AFSARMANESH, 2003):

- Agilidade: capacidade das empresas virtuais reagirem às mudanças;

- Papéis complementares: as empresas buscam uma complementaridade para que seja possível a participação em oportunidades competitivas e novos mercados;

- Incremento de dimensão: possibilidade de parcerias entre pequenas empresas "aumentar sua dimensão" e competir em mercados maiores;

- Compartilhamento de custos, riscos e recursos;

- Inovação: compartilhamento de idéias podem suscitar inovações.

Azevedo, A. (2000) complementa algumas características das empresas virtuais:

- Autonomia: as empresas são, em geral, independentes, podendo nomeadamente integrar

\footnotetext{
${ }^{1}$ Neste trabalho não será feita distinção entre os termos "organizações virtuais" ou "empresas virtuais". Para entendimento geral, adotou-se o termo empresas virtuais, nas demais citações ao longo do texto.
} 
outras empresas virtuais, ou encontrar-se integradas em estruturas de cooperação com contratos de longo prazo;

- Distribuição: as entidades que compõem a empresa virtual encontram-se fisicamente dispersas, eventualmente muito afastadas (por exemplo, em diferentes continentes);

- Configuração dinâmica: constituindo em geral uma aliança temporária, a empresa virtual tem subjacente um ciclo de vida que, para além do início e fim da própria empresa virtual, compreende a entrada e saída de novas entidades, de acordo com as oportunidades do mercado em que opera, originando-se assim atividades de reconfiguração;

- Redes de Informação: a emergência da empresa virtual como paradigma organizacional foi possível, em grande parte, ao desenvolvimento sem precedentes das tecnologias de informação e comunicação.

A gênese de uma empresa virtual está na capacidade para criar e estabelecer cooperações temporárias e aproveitar oportunidades de negócio efêmeras, tais que, uma empresa atuando de forma isolada, por incapacidade tecnológica ou limitação de recursos, não conseguiria aproveitar (AZEVEDO, A., 2000).

Resultante deste comportamento dinâmico, provocado pela necessidade de constante adaptação ao mercado, Goranson (1999) propôs um ciclo de vida estruturado em cinco fases principais e ilustrado na Figura 1:

Figura 1 Ciclo de vida de uma empresa virtual

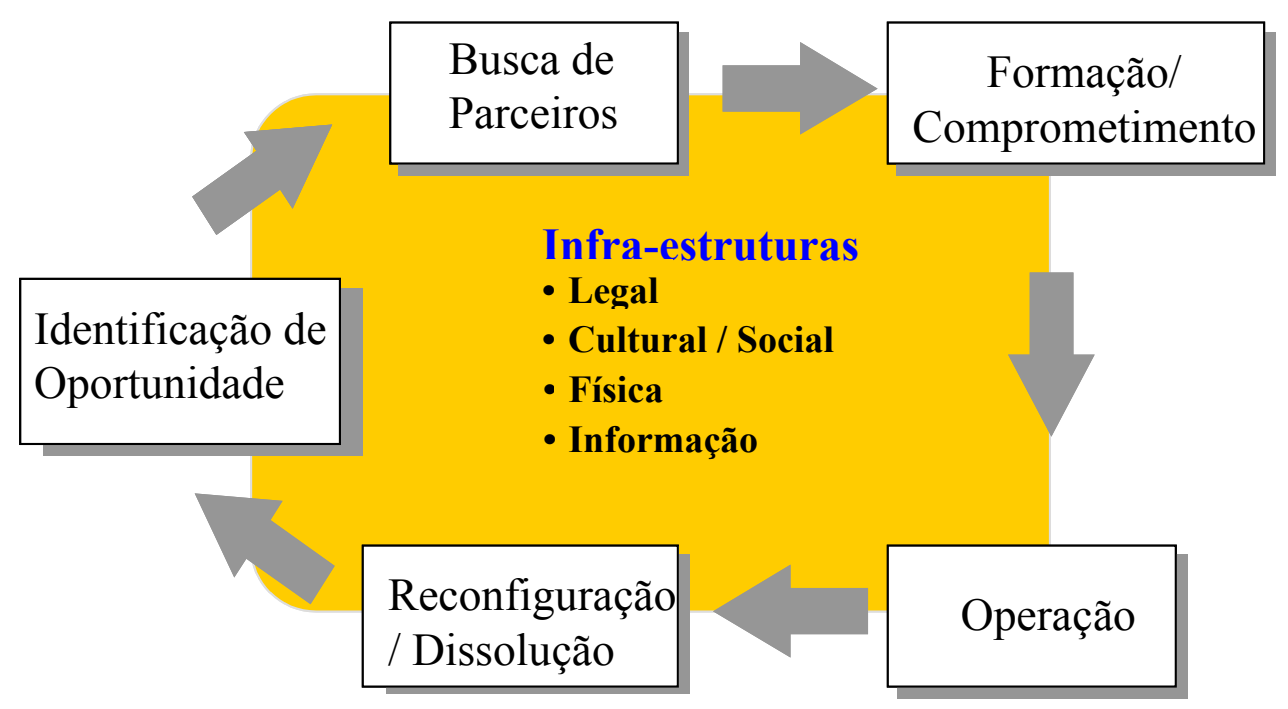

Fonte: Proposto a partir de Goranson (1999) 
Segundo Goranson (1999) apud Freitas (2005) as cinco fases podem ser descritas como:

- Identificação de Oportunidade: um líder em potencial ou um grupo de especialistas detêm a responsabilidade de identificar, refinar e/ou caracterizar a oportunidade de negócio;

- Busca de Parceiros: identificada a oportunidade, é necessário encontrar os parceiros adequados para atuar na rede;

- Formação: identificada a oportunidade e seus parceiros é necessário elaborar um business case detalhado;

- Operação: estabelecida a rede, esta deve ser operacionalizada, ou seja, colocada em funcionamento;

- Dissolução e Reconfiguração: em certo ponto pré-estabelecido, a oportunidade terá sido explorada integralmente ou necessitará ser modificada.

As quatro infra-estruturas definidas no modelo são (GORANSON, 1999 apud FREITAS, 2005):

- Informação: mecanismos usados para criar, administrar e comunicar informação;

- Social/Cultural: regras implícitas e explícitas e questões políticas existentes na organização. As regras de negócio associadas à supervisão são partes desta infraestrutura. A política, incluindo acordos trabalhistas e hábitos, é parte da cultura corporativa;

- Legal: Relaciona-se com processos que lidam com instrumentos legais. Internamente tais instrumentos são cláusulas contratuais; externamente eles são formados pelos códigos leis e regulamentações. Dentro desta questão, encontra-se o problema da supervisão da programação e controle de atividades. Redes de supervisão e papéis de decisão são incluídos;

- Física: relaciona-se com a fabricação, equipamentos, layout, transportes, manipulação ou quaisquer características físicas da rede.

É importante esclarecer que existem outras interpretações do ciclo de vida de empresas virtuais na literatura, a saber por exemplo: Fucks (1997), Merkle (1997), Zimmermman (1996), Camarinha-Matos e Afsarmanesh (1997) apud Pithon (2004).

\subsection{Modelagem de Redes}


As organizações bem sucedidas sempre tomam medidas que visam adaptar-se a seu ambiente. Fazem assim monitorando mudanças tecnológicas, avaliando os competidores, adaptando-se a legislação ou outros fatores que afetam as suas estratégias e a dos seus concorrentes. As mudanças na dinâmica do conhecimento implicam um imperativo claro: cada organização precisa embutir o gerenciamento das mudanças em sua própria estrutura. Cada organização deve se preparar para planejar o abandono, em vez de tentar prolongar a vida de um produto, política ou prática de sucesso; deve se dedicar à criação do novo. Em termos específicos: aperfeiçoamento contínuo de tudo aquilo que a organização faz, aprender a explorar seus conhecimentos, e inovar (Drucker, 1999, p. 60).

Segundo Freitas (2005), as empresas precisam de sistemas ágeis a mudanças constantes do ambiente do negócio para garantir que os sistemas cumpram sua finalidade. Os desenvolvedores devem possuir uma compreensão profunda sobre a organização, seus objetivos, metas e estratégias de mercado.

Para permitir a integração de empresas é necessário que todos os elementos que a compõem, sejam eles homens, máquinas e sistemas computacionais, entre outros, sejam capazes de trocar informações entre si numa profundidade além da simples troca física de dados. Isto passa necessariamente pelo desenvolvimento de uma visão holística dentro da empresa, isto é, o desenvolvimento de uma imagem única e integrada nas pessoas que fazem parte desta organização. É por meio da atuação de pessoas possuidoras desta imagem ampla e integrada e, portanto, capazes de considerar a interação entre múltiplos fatores, que se desenvolve e sedimenta a integração da manufatura. Um dos mecanismos que podem auxiliar as pessoas a obter esta imagem integrada da empresa são os modelos de empresa (AMARAL e ROZENFELD, 1999).

Modelos de empresa são representações de uma organização real que servem como uma referência comum para todos os seus membros, sejam eles pessoas, sistemas ou recursos. Este modelo forma uma infra-estrutura de comunicação que pode ter diversas aplicações. A partir do modelo de empresa qualquer pessoa pode adquirir uma visão geral sobre as operações, possibilitando análises, previsão de impactos das atividades, identificação de pontos de melhorias, entre outros, servindo, assim, como uma representação da visão holística. Com o apoio dos modelos de empresa é possível uma avaliação mais apurada do papel dos recursos nos processos de negócio e a análise e projeto da integração destes recursos com os demais (AMARAL e ROZENFELD, 1999).

Cada organização tem missão, objetivos e processos próprios e é importante dar atenção à modelagem desses itens. Alencar (1999) apud Pádua; Cazarini e Inamasu (2004) destaca os seguintes objetivos da Modelagem Organizacional:

- Fornecer um objeto, que seja uma representação compartilhável e reusável da cadeia de fornecimento de informação e conhecimento;

- Suportar tarefas da cadeia de fornecimento, pela habilitação de respostas a questionamentos, que não estão explicitamente representados no modelo; 
- Definir os objetos de maneira precisa, de forma que sejam consistentemente aplicados, por meio dos domínios e interpretados pelos usuários; e

- Suportar visualização do modelo, de forma intuitiva, simples e consistente.

Existem atualmente diversas propostas direcionadas à modelagem de empresas. Há princípios, etapas e uma grande quantidade de metodologias e ferramentas, também conhecidas como frameworks de modelagem. Apesar de todo este desenvolvimento e da importância que esta área vem recebendo dentro das organizações ainda persistem grandes barreiras para a aplicação destes modelos. Uma das principais barreiras é a complexidade gerada pela quantidade grande de elementos necessários para a representação destes tipos de sistemas (AMARAL e ROZENFELD, 1999).

Diversas metodologias podem ser utilizadas para desenvolver modelos de empresa, variando em níveis de sofisticação e abrangência. Na realidade um modelo pode ser desenvolvido desde a partir de uma simples linguagem gráfica reproduzida à mão até empregando frameworks sofisticados que empregam diferentes visões e modernos conceitos como orientação à objeto (AMARAL e ROZENFELD, 1999).

Amaral e Rozenfeld (1999), baseando-se no trabalho de Doumeingts; Vallespir e Chen (1995), classificaram dentre outros tipos de metodologias de modelagem o conceito de arquiteturas de referência, sendo esse um conjunto de modelos contemplando uma coleção invariante de building blocks (elementos fundamentais) a partir dos quais podem ser projetados ou representados todo um sistema integrado.

Segundo Freitas (2005) a metodologia Enterprise Knowledge Development (EKD) representa um arquitetura de referência interessante para a modelagem de redes de empresas. Assim, o tópico subseqüente procurou conceituar de maneira mais detalhada essa metodologia com o objetivo de servir de base teórica para a discussão que se segue neste trabalho.

\subsubsection{A metodologia EKD para Modelagem de Redes}

O EKD pode ser uma ferramenta apara a construção do modelo organizacional, pois é uma metodologia que fornece uma forma sistemática e controlada de analisar, entender, desenvolver e documentar uma organização e seus componentes, usando a Modelagem Organizacional. Tal metodologia tem como propósito descrever de maneira clara e não ambígua (NURCAN e ROLLAND, 2003):

- Como a organização funciona atualmente;

- Quais são os requisitos e as razões para a mudança; 
- Quais alternativas deveriam ser criadas para encontrar esses requisitos; e

- Quais são os critérios e argumentos para avaliação dessas alternativas.

Segundo Nurcan e Rolland (2003), o EKD é um método para documentação da empresa, seus objetivos, processos de negócios, e que auxilia a organização a desenvolver "conscientemente" procedimentos sistemáticos no processo de gestão de mudanças.

De acordo com Nurcan e Rolland (2003), o EKD pode ser visualizado em diversos níveis: Objetivos Organizacionais, Processos Organizacionais e Sistemas de informação.

Nesse contexto, o EKD auxilia o processo de conhecimento organizacional através de seus modelos que proporcionam várias visões (Figura 2) da organização:

Figura 2 Níveis de Modelagem Organizacional do EKD

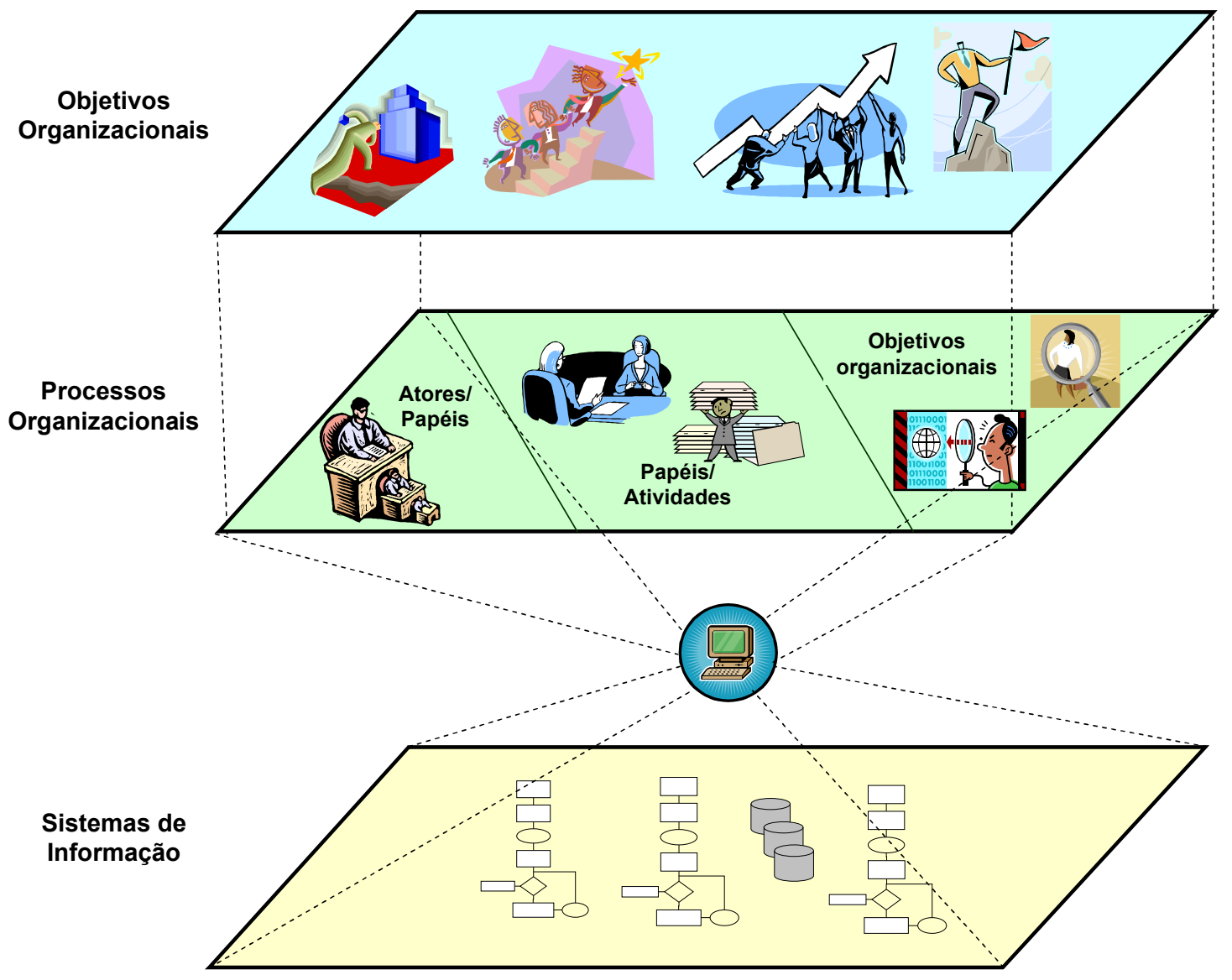

Fonte: Baseado em Nurcan e Rolland (2003).

Essa consideração sobre os níveis de modelagem no EKD será extremamente importante quando comentado sobre a proposição da arquitetura de referência, próximo item a ser discutido.

A metodologia EKD é constituída por modelos conceituais que examinam a organização e seus requisitos de um número de perspectivas inter-relacionadas. Esses modelos são abstrações do mundo básico. Para uma dada organização, esses modelos irão constituir coletivamente o Modelo 
Organizacional. Alguns desses modelos podem conter informações relevantes que apontam a necessidade de avaliação de alternativas de situações operacionais. Tais informações incluem critérios para avaliação, escolhas disponíveis, parâmetros de medidas e argumentos registrados a favor e contra escolhas (GANGA e CAZARINI, 2003).

Para uma dada empresa, eles constituirão coletivamente o modelo da empresa, cada um representando um aspecto da organização. De acordo com Bubenko et al. (1999) apud Pádua; Cazarini e Inamasu (2004, p.203) os tipos de submodelos e as questões que eles abordam são:

- Modelo de Objetivos: concentrado na descrição de idéias da organização. Descreve o que a organização e os empregados querem alcançar ou evitar, e quando;caso do uso de listas, deve-se usar o marcador que aparece no início desta frase;

- Modelo de Regras do Negócio: usado para definir e manter explicitamente regras do negócio formuladas e consistentes com o Modelo de Objetivos. Regras do Negócio podem ser vistas como operacionalização ou como limites dos objetivos;

- Modelo de Conceitos: Usado estritamente para definir "coisas" e "fenômenos" relacionados a outros modelos. Representa entidades organizacionais, atributos e relacionamentos. Entidades são usadas para definir mais estritamente expressões do Modelo de Objetivos, tanto quanto o conteúdo do conjunto de informação do Modelo de Processos do Negócio;

- Modelo de Processos do Negócio: usado para definir processos organizacionais, e a forma pela qual eles interagem e manuseiam a informação e os materiais. Um processo de negócio deve consumir as entradas (informação e/ou material) e produzir uma saída (informação e/ou material). Em geral o MPN é similar aos tradicionais modelos de diagramas de fluxo de dados (DFD);

- Modelo de Atores e Recursos: usado para descrever como diferentes atores e recursos se relacionam, e como eles são relacionados a componentes do Modelo de Objetivos e a componentes do Modelo do Processo do Negócio. Por exemplo: um ator pode ser responsável por um particular processo no MPN ou um ator pode buscar um particular objetivo no MO;

- Modelo de Requisitos e Componentes Técnicos: usado quando a proposta do EKD é ajudar a definir os requisitos para o desenvolvimento de um sistema de informação. Esse modelo direciona para o sistema técnico que é necessário para apoiar os objetivos, processos e atores da organização. Inicialmente, é necessário desenvolver um conjunto de requisitos de alto nível ou objetivos para o sistema de informação como um todo. Baseado nesses requisitos, o sistema de informação é estruturado em um número de subsistemas, ou componentes técnicos. O MCRT é uma tentativa inicial de se definir toda a estrutura e propriedades do sistema de informação, para apoiar as atividades do negócio, como definido no MPN (BUBENKO et al.,1999 apud PÁDUA; CAZARINI e INAMASU, 2004, p.203); 
O EKD assim, torna-se um referencial comum entre as áreas da empresa, independente de tecnologia, a fim de permitir a sua execução em diversas plataformas da tecnologia e, portanto, independente das mudanças tecnológicas. Somente a situação e o contexto em que a empresa existe e opera podem determinar as mudanças. Possibilita avaliar as opções, e documentar todos os aspectos intangíveis. A proposta do EKD aproxima as arquiteturas de referência da tratativa da tecnologia como conhecimento e não como informação (GUERRINI, 2006).

\section{Arquiteturas para redes de cooperação em sistemas produtivos dinâmicos}

As empresas virtuais, novas formas de organização fortemente baseadas na cooperação e duração muito variável, pressupõem a existência de um conjunto de instrumentos de apoio à gestão, com base sem tecnologias de informação e comunicação, que suportem adequadamente o seu ciclo de vida. Mas o que se constata atualmente é que os requisitos destas novas formas de organização, seja tanto à nível funcional quanto à infra-estrutura tecnológica, ainda não são cabalmente satisfeitos pelas soluções disponíveis comercialmente [...] (AZEVEDO, A., 2000).

“As fases de configuração, reconfiguração e operação parecem ser as mais exigentes e para as quais há atualmente, menos soluções disponíveis. Em particular, a configuração e a reconfiguração necessitam de instrumentos para mapear (grifo nosso), de forma eficiente, os sistemas e processos físicos produtivos em agentes de informação [...]” (AZEVEDO, A., 2000, p. 217).

No contexto citado se justifica a proposição de arquiteturas de referência para redes de cooperação entre empresas, nomeadamente as "empresas virtuais".

A arquitetura de referência é uma estrutura ou framework que guia durante todo o projeto de desenho e a implementação de um sistema de empresas integradas no sentido de uma metodologia estrutural, a formalização das operações e as ferramentas de suporte. Ela deve guiar o desenvolvimento e a aplicação de todas as disciplinas envolvidas em um projeto de integração de empresas, sistematicamente modelando todas as partes do ciclo de vida (CHALMETA; CAMPOS e GRANGEL, 2001).

Uma arquitetura de referência tem como objetivo desenvolver uma infra-estrutura de informação integrada que comunica e coordena os diferentes dispositivos tecnológicos que geram, processam, distribuem e fornecem informação. Elas devem ser orientadas para a integração de toda a organização, considerando não apenas os aspectos tecnológicos do sistema, mas também os aspectos econômico, social e humano (CHALMETA; CAMPOS e GRANGEL, 2001).

Esta consideração é interessante, por relacionar o EKD como uma arquitetura de referência, já que o mesmo trabalha em sua metodologia não só com aspectos técnicos, mas também outros 
aspectos relacionados a organização (processos, estratégia, gestão de mudanças, pessoas) e os requisitos técnicos de sistemas de informação.

Como citado anteriormente, Amaral e Rozenfeld (1999), conceituam arquitetura de referência como sendo esse um conjunto de modelos que contemplam uma coleção invariante de building blocks (elementos fundamentais) a partir dos quais podem ser projetados ou representados todo um sistema integrado.

Esse sistema integrado pode ser extrapolado como uma rede de cooperação entre empresas, mas especificamente uma empresa virtual e os agentes que compõem a mesma.

A arquitetura de referência é um conjunto de elementos relacionados entre si que formam um arcabouço para uma determinada finalidade (VERNADAT, 1996). No caso de uma empresa virtual, a exploração temporária de uma oportunidade de mercado.

Existem inúmeras arquiteturas de referência na literatura. Contudo Freitas (2005) faz referência em seu trabalho as mais representativas segundo o escopo de modelagem empresarial disponível, a saber: ISO (Intenational Standart Organization) work; CEN (Comitê Europeu para Padronização)-ENV (European Prestandart) 40 003; ARIS (Arquitetura para Sistemas de Informação Integrada); CIMOSA (Sistema Europeu de Arquitetura Aberta para Manufatura Integrada por Computador); PERA (Purdue Enterprise Reference Architeture) e o próprio EKD, discutido anteriormente.

Guerrini (2006) cita ainda outras arquiteturas de referência voltadas à redes de empresas: AMEF (Agile Manufacturing Enterprise Forum); AAMRC (Agile Aerospace Manufacturing Research Center) e a Arquitetura ARDIN, proposto por Chalmeta; Campos e Grangel (2001) principalmente aplicado à pequenas e médias empresas.

\subsection{Proposta de um Modelo Genérico de Arquitetura para Redes de Cooperação em Sistemas Produtivos Dinâmicos a partir do EKD}

O objetivo desta seção não é propor o estado da arte em arquitetura de referências para redes de cooperação nem discutir peculiaridades de outros modelos já desenvolvidos.

Pretende-se aqui lançar mão de conceitos discutidos anteriormente e formar um arcabouço conceitual dos parâmetros que devem ser levados em consideração num projeto de arquitetura de referência para redes de cooperação.

Assim, o modelo genérico proposto para arquitetura de redes de Cooperação pode ser observado na figura 3. 
Figura 4 Visão geral de uma arquitetura para Redes de Cooperação

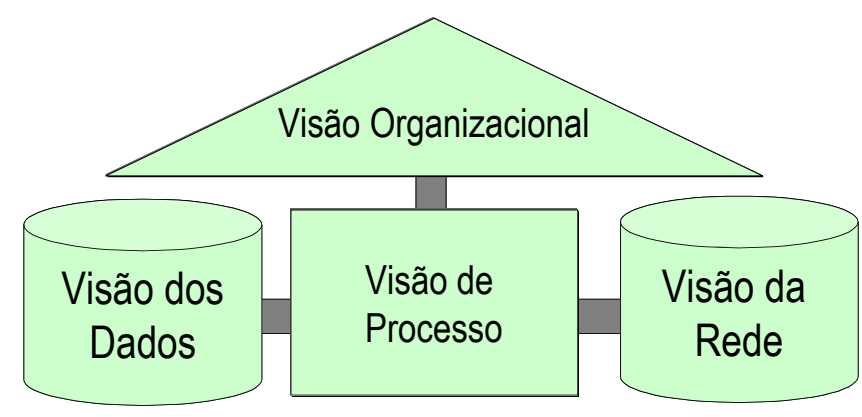

Fonte: Proposta pelo autor

Num contexto mais amplo a Visão Organizacional está relacionada questões mais estratégicas da rede, como definição de metas e objetivos, estabelecimento do ciclo de vida da rede, indicadores de desempenho dessas fases e própria definição dos papéis e responsabilidades de cada agente que forma a rede, ou seja, quem faz o quê, e também quem poderá exercer a governança. As três visões seguintes representariam características mais operacionais da rede. No entanto esse comentário não pode conjecturar-se como uma definição rígida, já que essas visões estão interrelacionadas, e muitas vezes podem até sobrepor-se, como é o caso da Visão da Rede, que poder adquirir características mais estratégicas do que operacionais na caracterização das relações interorganizacionais. Nesse sentido a Visão dos Dados contém a descrição semântica dos dados, representando assim toda a estrutura de informação pela Rede. A Visão de Processo representa a modelagem dos processos que se formam através da interação entre os agentes que compõem a rede, além de relacionar-se com as demais visões e finalmente, a Visão da Rede contém os modelos que definem a morfologia e tipologia da rede, e outras variáveis de caracterização da rede, como ambiente e identificação de competências.

A fim de representar melhor esse modelo genérico de arquitetura de referência para redes, foi proposta uma visão em camadas para essa arquitetura de referência como pode ser observado na figura 5 . 
Figura 5 Camadas de uma Arquitetura de Referência genérica para redes de Cooperação

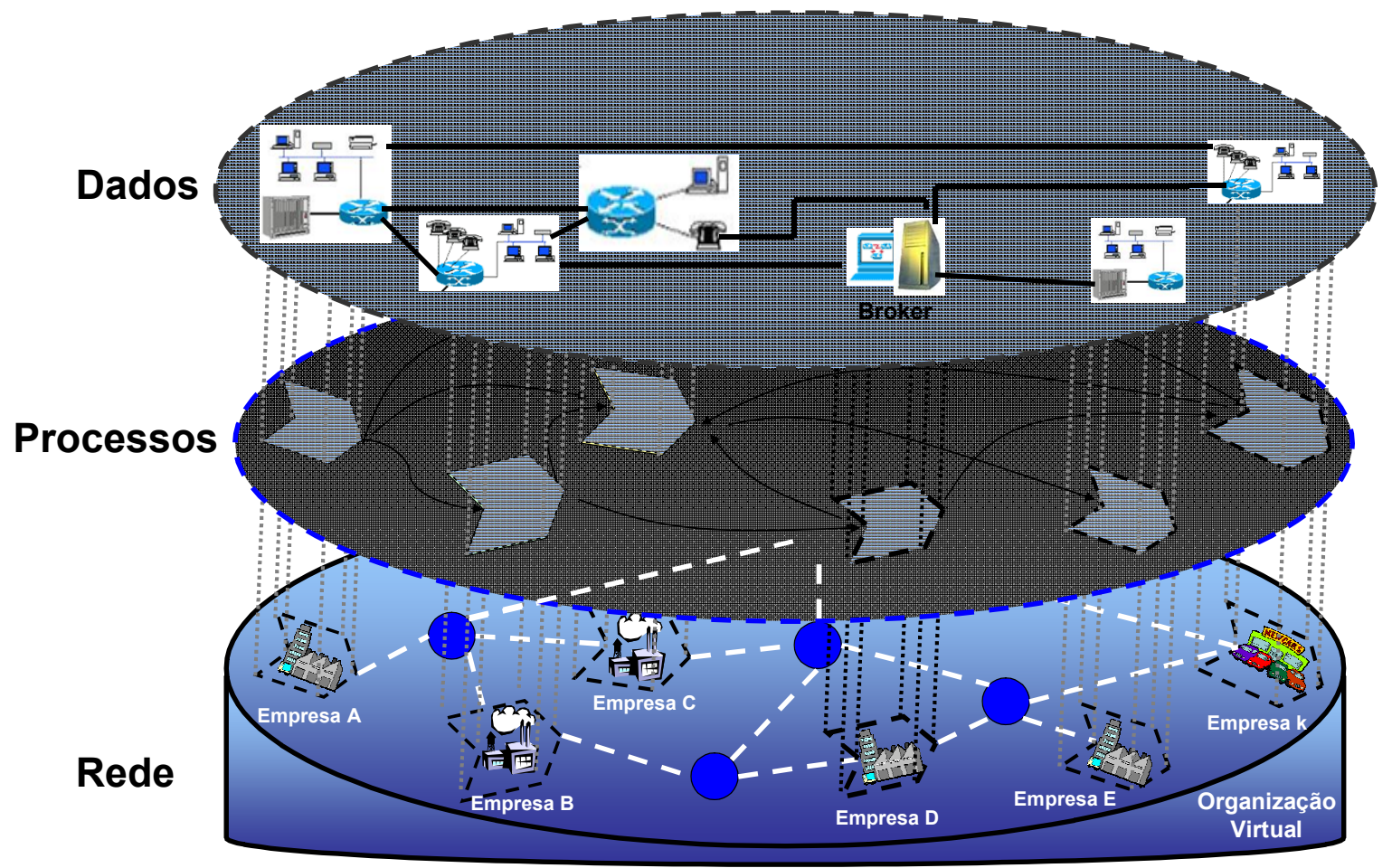

Fonte: Proposta pelo autor

A interpretação das visões da arquitetura genérica para redes pode ser relacionada aos vários níveis do EKD, como pode ser observado na figura 2. No EKD a definição dos objetivos organizacionais estratégicos acabava direcionando a formação dos processos de negócios que por sua vez davam parâmetros, através da modelagem dos mesmos, para proposição de um modelo de requisitos e componentes técnicos que mais tarde resultaria em um sistema de informação. Essa estrutura sistêmica e dinâmica de gestão do conhecimento organizacional forneceria subsídios técnicos, de gestão e informacionais para a empresa interagir tanto com seu ambiente externo quanto interno e reagir eficazmente às mudanças constantes, podendo assim elevar sua competitividade.

Para o modelo genérico de arquitetura para redes de cooperação proposto observa-se a mesma relação lógica nas demais fases do seu ciclo de vida. A formação da rede e escolha dos parceiros, a partir da identificação de oportunidades estratégicas define os objetivos desse interrelacionamento e os papéis de cada agente nessa rede. Na fase de operação é necessário que se conheçam os processos de negócios que são formados pela interação dos agentes nessa rede de cooperação. È necessário ainda que esses processos de negócios sejam suportados por tecnologias de informação, uma condição essencial para facilitar a interação entre esses agentes, segundo Cao e Dowlatshahi (2005). A agilidade da rede em uma empresa virtual seria obtida através da redução do controle de gestão hierárquica, agrupamento de trabalhadores em equipes e seu fortalecimento para o processo de tomada de decisão. 
Em relação à definição dessa plataforma de comunicação da rede, Camarinha-Matos e Afsarmanesh (1999) revelam que existe grande problema na configuração dessa plataforma de informação, devido a grande diversidade de padrões tecnológicos entre as empresas que compõem a rede.

Em relação aos modelos do EKD e o modelo genérico de arquitetura de referência para redes de cooperação podem ser feitas as seguintes considerações:

O Modelo de Objetivos e o Modelo de Atores e Recursos estão relacionados à Visão organizacional, seja na definição dos próprios objetivos da rede, bem como o desdobramento em sub-objetivos para cada agente que compõe a mesma, além é claro da definição dos papéis e responsabilidades de cada membro, descrição da estrutura organizacional entre outros. O Modelo de Processos de Negócios do EKD está relacionado à Visão de Processos da Arquitetura. Outros modelos do EKD, como por exemplo, o Modelo de Regras de Negócio apóiam outros modelos. Mais especificamente o modelo de regras de negócio se faz extremamente necessário principalmente na fase de operação da rede, quando, por exemplo, na formalização de contratos e acordos de cooperação, a questão da segurança em relação à propriedade de conhecimento por determinados agentes, etc. Esse modelo também é importante na restrição de alguns objetivos que pareçam conflitantes na formação da rede. O Modelo de Requisitos e Componentes Técnicos está relacionado à Visão de Dados do modelo de Arquitetura de Referência proposto. Ele poderá ser extremamente útil na determinação de uma mesma plataforma de comunicação entre as empresas que compõem a rede. O Modelo de Conceitos de maneira geral apóia todos os outros modelos no sentido de criar um "banco de conceitos", que podem ser os contratos, legislações entre outras informações importantes no entendimento dos outros modelos ou visões.

Por fim, a Visão da rede está relacionada à caracterização da própria rede em si, sua tipologia, morfologia, ambiente e identificação de competências.

\section{Considerações Finais}

No atual panorama de competitividade mundial a agilidade e a flexibilidade tornaram-se essenciais para as organizações. Responder eficazmente ás solicitações dos clientes tornou-se fundamental na determinação de vantagens competitivas sustentáveis. Nesse sentido a pressão por menores ciclos produtivos, menores tempos de lançamentos de produtos, maior variedade do mix de produtos e inovações tecnológicas, entre outros, tornou imperativo a busca ou desenvolvimento de novas configurações organizacionais que pudessem suportar esse cenário complexo e dinâmico.

As redes de cooperação, principalmente de pequenas e médias empresas, tornaram-se então uma alternativa viável para estas se manterem competitivas no mercado. A palavra da ordem 
deixava de ser "competir" para "cooperar". No entanto, uma cooperação que suscite mútuos benefícios necessita de toda uma estrutura de coordenação, que promova uma confiança tal entre os agentes envolvidos, a fim de se evitar um possível ambiente de "coopetição".

Uma arquitetura de referência pode fornecer elementos para a integração e coordenação entre empresas. Ele pode dar suporte a todo o ciclo de vida de uma rede de cooperação. Numa empresa virtual, por exemplo, ela pode auxiliar fortemente as fases de formação, operação e reconfiguração/dissolução da rede, seja fornecendo subsídios para identificação de competências/parceiros, gestão durante a operação através da coordenação dos agentes via tecnologias de informação e comunicação, definição de indicadores de desempenho da rede, entre outros mais.

Em relação ao modelo genérico proposto para uma arquitetura de referência, constata-se que apesar da grande relevância em relacioná-lo à metodologia EKD, ainda são necessários muitos refinamentos e considerações estruturais para validação do mesmo. Assim, a grande contribuição do mesmo se resume a menção de uma série de características de redes de cooperação, no caso, empresas virtuais, que podem vir a ser apoiadas pela metodologia EKD.

Como conclusão geral, observa-se a grande aplicabilidade da metodologia EKD como uma Arquitetura de Referência ou até mesmo suportando outra arquitetura comercial existente.

\begin{abstract}
The pressure for shorter productive cycles, shorter development of products cycles, technological innovations and larger products mix, became imperative the search or development by news organizationals configurations that could support the current complex and dynamic environment. The Networks Organizations, mainly in small and medium sized firms, had become than a viable alternative for these to keep competitive in the market. In this direction, a reference architecture can supply elements to the integration and coordination among companies. This work aims to present the relative concepts to the Architecture of Reference for Networks Organizations consisting in dynamic productive systems as form to co-ordinate resources, capabilities and competences. It is also aims to consider a generic and conceptual model of reference architecture and to relate it approach EKD (Enterprise Knowledge Development). As conclusion, it is observed great applicability of methodology EKD as an Architecture of Reference or even though supporting another existing commercial architecture.
\end{abstract}

Key-words: network organization; network modeling; architecture's reference; virtual enterprises; enterprise knowledge development (ekd).

\title{
Referências
}

ALTER, C.; HAGE, J. Organizations working together. Newbury Park, CA: Sage, 1993.

ALENCAR, F. M. R. Mapeando a Modelagem Organizacional em especificações precisas. 1999. 304 f. Tese (Doutorado) - Centro de Informática, Universidade Federal de Pernambuco, Recife, 1999. 
AMARAL, D. C.; ROZENFELD, H. Modelagem de Empresas. (1999) Disponível em: http://www.numa.org.br/conhecimentos/conhecimentos_port/pag_conhec/Modelagemv1.html.Acesso em 05 Jan. 2006.

AMATO NETO, J. de. Redes dinâmicas de cooperação e organizações virtuais. In: AMATO NETO, J. (Org.) Redes entre organizações: domínio do conhecimento e da eficácia operacional.(2005). São Paulo: Atlas, 2005.

AZEVEDO, A. L. A emergência da empresa virtual e os requisitos para os sistemas de informação. Gestão \& Produção. v.7, n.3, p. 208-225, dez., 2000.

AZEVEDO, P. F. de. Nova Economia Institucional: referencial geral e aplicações para a agricultura. Agricultura São Paulo, SP, p. 33-52., 2000.

BRITTO, J. Elementos estruturais e conformação interna das redes de firmas: desdobramentos metodológicos, analíticos e empíricos: uma síntese da literatura. Niterói, Universidade Federal Fluminense, 2002.

BUBENKO JR.; J.A.; STIRNA, J.; BRASH, D. EKD user guide, Dpt of computer and systems sciences. Stockholm: Royal Institute of Technology, 1999.

CAMARINHA-MATOS, L. M.; AFSARMANESH, H. Elements of a base VE infrastructure. Computers in Industry, 51, 139-163., 2003.

cross'

CAMARINHA-MATOS, L. M. E AFSARMANESH H. Virtual Enterprises: Life Cycle Aupporting Tools and Technologies. In A. Kusiak (Ed.), Handbook of Life Cycle Engineering: Concepts, models and technologies, pp.535571: Kluwer Academic Publisher, 1997.

CAO, Q.; DOWLATSHAHI, S. The impact of alignment between virtual enterprise and information technology on business performance in an agile manufacturing environment. Journal of Operations Management, 23, 531-550, 2005.

cross ${ }^{\text {ref }}$

CARVALHO, M. M. de. Relações entre empresas, competências coletivas e tipos de governança em clusters de alta tecnologia do Estado de São Paulo. In: AMATO NETO, J. (Org.) Redes entre organizações: domínio do conhecimento e da eficácia operacional. São Paulo: Atlas, 2005.

CHALMETA,R.; CAMPOS, C.; GRANGEL, R. References architetures for enterprise integration. The Journal of Systems and Software, 57, 175-191, 2001.

COOPER, R.; SLAGMULDER, R. Inter-organization Cost management and Relation Context. Accounting, Organization and Society, v. 29, p. 1-26, 2004.

cross'

CUNHA, C. R. da.; MELO, M. C. de O. A confiança nos relacionamentos interorganizacionais: o campo da biotecnologia em análise. RAE-eletrônica, v.5, n.2, Art 18, jul./dez, 2006.

DODGSON, M. Learning, trust and technological collaboration. Human relations, v. 46, n. 1, p. 77-95, 1993.

cross ${ }^{\text {ref }}$

DOUMEINGTS, G; VALLESPIR, B.; CHEN, D. Methodologies for designing CIM systems: a survey. Computers in Industry, v.25, n.3. p.263-280, 1995.

cross ${ }^{\text {ref }}$

DRUCKER, P. Administrando em tempos de grandes mudanças. São Paulo: Pioneira; Publifolha, 1999,286 p.

FLEURY, A. C. C.; FLEURY, M. T. L. Estratégias empresariais e formação de competências: um quebra cabeça caleidoscópico da indústria brasileira. 2. ed. São Paulo: Atlas, 2000.

FREITAS, J. B. de. Formação e gerência de redes de empresas: requisitos para adequação do planejamento e controle de produção - estudo de caso em obras de construção civil de grande porte. Dissertação (Mestrado). 2005. Programa de Pós Graduação em Engenharia de Produção, Escola de Engenharia de São Carlos, Universidade de São Paulo, 140p. 
FUCKS, M. Design and implementation of Value Systems: The Lifecycle Perspective. Institute for Technology Mabagement, University of St. Gallen, Switerland, $1997 . \quad$ disponível em http://www.nectar.org/update/proceedings/97082101/fuchs/index.html.

GANGA, G. M. D.; CAZARINI, E. W. Modelo de objetivos e regras do negócio da abordagem ekd: estudo de caso em clínica odontológica. XXIII Encontro Nacional de Engenharia de Produção (ENEGEP), Ouro Preto, Anais... 2003.

GOEDERT, A. R. Governança em rede de desenvolvimento e a experiência em Santa Catarina. Tese (Doutorado) em Engenharia de Produção, Universidade Federal de Santa Catarina, Florianópolis, 446p., 2005.

GORANSON, H. T.The agile virtual enterprise: cases, metrics, tools. Quorum Books, 1999.

GRANDORI, A.; SODA, G. Inter-firm networks: antecedents, mechanisms and forms. Organizational Studies, v. 16, n. 2, p. $183-214,1995$.

cross ${ }^{\text {ref }}$

GUERRINI, F. M. Arquitetura para redes de cooperação em sistemas produtivos dinâmicos. Dinâmica do Curso. Material de apoio à disciplina SEP 5791 (Arquitetura para redes de cooperação em sistemas produtivos dinâmicos), Programa de Pós Graduação em Engenharia de Produção, Escola de Engenharia de São Carlos, Universidade de São Paulo, 2006.

HOLLINGSWORTH; LINDBERG. The governance of the american economy: markets, clans, hierarchies and associative behavior. In: STREECK e SCHIMITTER. Private interest government. New York: University Press, 1986.

HUMPHREY, J.; SCHIMITZ, H. Governance and upgrading: linking industrial clusters and global value chain research. IDS Working Paper 120, p. 1-37, 2000

JESSOP, B.The rise of governance and the risks of failure: the case of economic development. International Social Science Journal, n. 155, p. 29-45, 1998.

cross ${ }^{\text {ref }}$

LEON, M. E. Uma análise de redes de cooperação das pequenas e médias empresas do setor das telecomunicações. 1998. Dissertação (Mestrado), Engenharia de Produção da Escola Politécnica da Universidade de São Paulo, 1998.

MANUAL DE OSLO. Manual de Oslo: proposta de diretrizes para coleta e interpretação de dados sobre inovação tecnológica. Brasília, OCDE, Finep, 2004.

MERKLE, M. Virtual Organizations - how quality management paves the way for it. Institute for Techology Management, University of St. Gallen, Switerland, 1997.

NOOTEBOOM, B. Innovation and inter-firm linkages: new implications for policy. Research Policy, 28, 793-805, 1999.

cross ${ }^{\text {ref }}$

NOOTEBOOM, B. Interfirms collaboration, networks and strategy: an integrated approach. The Hague, 2003.

NURCAN, S.; ROLLAND, C. A multi-method for defining the organizational change. Information and Software Tecnology, 45, p. 61-82, 2003.

cross ${ }^{\text {ref }}$

OLAVE, M. E. L.; AMATO NETO, J. Redes de cooperação produtiva: uma estratégia de competitividade e sobrevivência para pequenas e médias empresas. Gestão \& Produção. V.8, n.3, p. 289-303, dez, 2001.

OLIVER, C.. Determinants of interorganizational relationships: integration and future directions. Academy of Management Review. v. 15. n. 2, p. 241-265, 1990.

cross ref 
PÁDUA, S. I. D. de; CAZARINI, E. W.; INAMASU, R. Y. Modelagem organizacional: captura dos requisitos organizacionais no desenvolvimento de sistemas de informação. Gestão \& Produção, v.11, n.2, p. 197-209, mai-ago., 2004.

PITHON, A. J. C. Projeto Organizacional para a engenharia concorrente no âmbito das empresas virtuais. $316 \mathrm{p}$. Tese (Doutorado).Engenharia de Produção e Sistemas. Universidade do Minho, Guimarães, Portugal, 2004.

POWELL, W. W. Neither market nor hierarchy: network forms of organization. Research in Organization Behavior, vol. 12, p. 295-336., 1990.

RIBAUlT, M.; MARTINET, B.; LEBIDOIS, D. A gestão das tecnologias. Coleção Gestão \& Inovação. Lisboa, Publicações Dom Quixote, 1995.

RING, P. S.; VAN de VEN, A. H. Developmental process of cooperative interorganizational relationships. Academy of Management Review , v. 19, n. 1, p. 90-118, 1994.

cross ${ }^{\text {ref }}$

RYCROFT, R. W.; KASH, D. E. Self-organization networks: implications for globalization. Technovation, $24,187-$ 197,2004

cross'

SACOMANO NETO, M.; TRUZZI, O. M. S. Perspectivas contemporâneas em análise organizacional. Gestão \& Produção, v.9, n.1, p. 32-44, abr., 2002.

SACOMANO NETO, M.; TRUZZI, O. M. S. Configurações estruturais e relacionais de rede de fornecedores: uma resenha compreensiva. RAE-USP, v.39, n.3, jul./ago./set., p. 255-263, 2004.

VERNADAT, F. B. Enterprise Modeling and Integration: principles and applications. New York: Champman \& Hall, London, 1996.

WILLIAMSON, O. E. The economic institutions of capitalism. London: Free Press, 1985. 450p.

ZIMMERMMAN, F. Structural and Manageral Aspects of Virtual Enterprises. University of Bamberg, Business Information Systems Department, 1996, disponível em http://www.seda.sowi.unibamberg.de/persons/zimmermann.html.

\section{Dados dos autores:}

Nome completo: Gilberto Miller Devós Ganga

Filiação institucional: Universidade Estadual Paulista (UNESP)

Departamento: Administração

Função ou cargo ocupado: Pesquisador do GMME - Grupo de Pesquisa em Gestão da Melhoria e Mudança Empresarial, Pesquisador da CEPEAGRO (Centro de Pesquisa em Administração e Agronegócios) e Professor Assistente do curso de Administração de Empresas do Campus Experimental de Tupã, UNESP.

Endereço completo para correspondência (bairro, cidade, estado, país e CEP): Av. Domingos da Costa Lopes, 780; CEP 17602-496 - Tupã, SP;

Telefones para contato: (14) 3404-4200

e-mail: giba@tupa.unesp.br

Nome completo: Fábio Müller Guerrini 
Filiação institucional: Universidade de São Paulo (USP) - Escola de Engenharia de São Carlos (EESC)

Departamento: Engenharia de Produção

Função ou cargo ocupado: Pesquisador do AR-C - Grupo de Pesquisa em Arquitetura Organizacional para Formação e Gerência de Redes de Cooperação entre Empresas e Professor Associado do Programa de Pós Graduação em Engenharia de Produção da Escola de Engenharia de São Carlos (EESC), Universidade de São Paulo (USP).

Endereço completo para correspondência (bairro, cidade, estado, país e CEP): Avenida Trabalhador São Carlense, 400, São Carlos-SP, Brasil.

Telefones para contato: (16) 3373-9428

e-mail: guerrini@sc.usp.br

Nome completo: Luiz Cesar Ribeiro Carpinetti

Filiação institucional: Universidade de São Paulo (USP) - Escola de Engenharia de São Carlos (EESC)

Departamento: Engenharia de Produção

Função ou cargo ocupado: Pesquisador do GMME - Grupo de Pesquisa em Gestão da Melhoria e Mudança Empresarial e Professor Associado do Programa de Pós Graduação em Engenharia de Produção da Escola de Engenharia de São Carlos (EESC), Universidade de São Paulo (USP).

Endereço completo para correspondência (bairro, cidade, estado, país e CEP): Avenida Trabalhador São Carlense, 400, São Carlos-SP, Brasil.

Telefones para contato: (16) 3373-9428

e-mail: carpinet@sc.usp.br 